\title{
ACIDENTES DE TRABALHO AFETANDO A COLUNA VERTEBRAL: UM ESTUDO REALIZADO COM TRABALHADORES DE ENFERMAGEM DE UM HOSPITAL UNIVERSITÁRIO*
}

\author{
Neusa Maria Costa Alexandre** \\ Maria Cecilia Cardoso Benatti***
}

ALEXANDRE, N.M.C.; BENATTI, M.C.C. Acidentes de trabalho afetando a coluna vertebral: um estudo realizado com trabalhadores de enfermagem de um hospital universitário. Rev.latino-am.enfermagem, Ribeirão Preto, v. 6, n. 2, p. 65-72, abril 1998.

Com o objetivo de avaliar determinadas características da ocorrência de acidentes do trabalho afetando a coluna vertebral em trabalhadores de enfermagem de um hospital universitário, entrevistou-se todos os funcionários que sofreram esse tipo de acidente em um periodo de seis meses. Esses trabalhadores eram basicamente do sexo feminino e tinham uma idade média de 43,2 anos. Não houve uma diferença estatística significante que demonstrasse um predominio de acidentes entre os turnos de trabalho e entre as diferentes categorias de enfermagem envolvidas. A maioria dos acidentes ocorreu na própria unidade de trabalho, quando esses trabalhadores estavam movimentando ou transportando pacientes e equipamentos e por quedas devido ao piso escorregadio. As regiões mais atingidas da coluna vertebral foram a lombar e a cervical e a maior parte dos entrevistados afirmou que já tinha tido algum tipo de problema na região dorsal.

UNITERMOS: acidente do trabalho, trabalhadores de enfermagem, lesões nas costas

\section{1 - INTRODUÇÃO}

A questão da saúde dos trabalhadores de hospitais tem, cada vez mais, preocupado estudiosos de todo o mundo. Atualmente, sabe-se que o pessoal de saúde está sujeito a pelo menos quatro tipos de riscos ocupacionais: biológicos, químicos, físicos e psicossociais ${ }^{27,48}$.

Dos grupos ocupacionais sujeitos a estes riscos, a Enfermagem destaca-se como um mais afetados pelos acidentes do trabalho ${ }^{27,40}$. É importante destacar que acidente do trabalho, do ponto de vista legal, "é aquele que ocorre pelo exercício do trabalho a serviço da empresa, provocando lesão corporal ou perturbação funcional que causa a morte ou a perda ou redução, permanente ou temporária, da capacidade para o trabalho" 28 .

Pesquisas que enfocam os acidentes do trabalho em trabalhadores de enfermagem, demonstram que os acidentes mais frequentes são as lesões pérfuro-cortantes seguidos pelos ferimentos que comprometem o sistema osteoarticular, advindos principalmente de contusões e torções ${ }^{38,39,41}$. Entre esses, destacam-se as lesões que comprometem a coluna vertebral.

Estas afecções vêm despertando a atenção de especialistas em saúde ocupacional por serem uma das mais importantes causas de morbidade e incapacidade de adultos ${ }^{20,31}$. Estudos que comparam os diversos grupos ocupacionais que trabalham em hospitais, indicam que os trabalhadores de enfermagem são os que mais são acometidos por lesões nas costas $5,10,16,37$.

Para muitos autores, os trabalhadores de enfermagem são especialmente suscetíveis a lesões nas costas pelo fato de terem que movimentar e transportar pacientes regularmente ${ }^{22,29,36,45}$. VIDEMAN ${ }^{46}$ destaca que essas lesões são particularmente pouco documentadas. Na literatura brasileira não existem trabalhos que abordem especificamente esse tema.

Dessa forma, o presente estudo tem por objetivo avaliar determinadas características da ocorrência de acidentes do trabalho relacionados com a coluna vertebral entre os trabalhadores de enfermagem de um hospital universitário.

\footnotetext{
* Trabalho apresentado no 25 th International Congress on Occupational Health - Estocolmo - 1996

** Professor Assistente Doutor do Departamento de Enfermagem da FCM - UNICAMP

*** Professor Assistente do Departamento de Enfermagem da FCM - UNICAMP
} 


\section{2 - METODOLOGIA}

\section{1 - População Estudada}

A população foi composta por trabalhadores de enfermagem (enfermeiros, técnicos de enfermagem, auxiliares de enfermagem e atendentes de enfermagem) de um hospital geral universitário com 403 leitos. A parcela da população estudada compreendeu todos os trabalhadores de enfermagem que sofreram acidentes do trabalho relacionados com a coluna vertebral durante um período de seis meses consecutivos (janeiro a junho de 1995).

\section{2 - Instrumento de Coleta de Dados}

Optou-se por uma entrevista, feita através de uma ficha com um roteiro pré-fixado, contendo questões fechadas e abertas.

Introdutoriamente, o formulário contém uma identificação com dados sobre as seguintes características do pessoal de enfermagem: idade, sexo, estado civil e categoria profissional. A seguir, apresenta uma parte com questões relacionadas especificamente com o acidente que causou a lesão nas costas, que compreende: turno de trabalho, local de ocorrência, causas, regiões da coluna vertebral mais atingidas e queixas anteriores de dores ou outras afecções relacionadas com a coluna vertebral.

Realizou-se um plano piloto e depois da aplicação deste as fichas foram revisadas e modificadas.

\section{3 - Coleta de Dados}

Os dados foram coletados por uma das autoras através da aplicação do formulário ao pessoal de enfermagem acidentado no período de janeiro a junho de 1995, à medida que os acidentes foram ocorrendo. Estes funcionários foram entrevistados nos seus respectivos locais de trabalho.

A relação destes funcionários era elaborada pelas diretoras de enfermagem, sendo registrada em uma ficha especificamente elaborada com esse objetivo. Estas diretoras foram previamente orientadas sobre a finalidade do estudo.

\section{4 - Análise dos Dados}

$\mathrm{Na}$ análise dos dados referentes ao sexo, turno de trabalho e categoria profissional foram utilizados os testes Qui-quadrado e Exato de Fisher.

\section{3 - RESULTADOS E DISCUSSÃO}

Inicialmente é importante destacar que, dos 1218 trabalhadores de enfermagem do hospital em estudo, 100 $(8,2 \%)$ sofreram algum tipo de acidente do trabalho em um período de seis meses. Desses 100, 20 (20\%) estavam relacionados com lesões na coluna vertebral. Ressalta-se também que dos 1.218 trabalhadores, 1.097 (90\%) eram do sexo feminino e 121 (10\%) do sexo masculino. Em relação aos 100 acidentados, observou-se esse mesmo contingente de trabalhadores do sexo feminino $(90 \%)$ e do sexo masculino (10\%).

No presente estudo foi entrevistado um total de 20 pessoas, sendo 19 (95\%) do sexo feminino e 1 (5\%) do sexo masculino. Quanto ao estado civil, pode-se observar que a maioria dos acidentados eram casados (Figura 1). A nível exploratório verificou-se através do teste Exato de Fisher que não existe evidência de associação entre o tipo de acidente de trabalho (coluna/ não coluna) e o sexo do trabalhador $(\mathrm{p}=0,681)$.

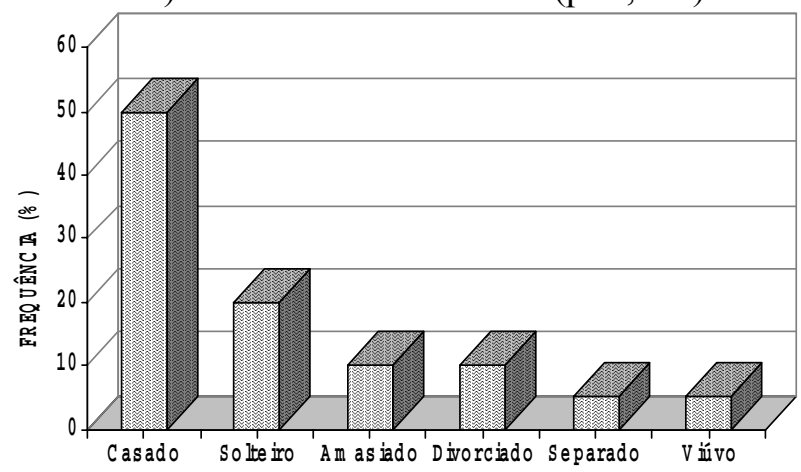

Figura 1 - Distribuição de trabalhadores de enfermagem que sofreram acidente de trabalho relacionado com a coluna vertebral segundo o estado civil. Campinas, 1995

Considerando que a maior parte dessas pessoas são do sexo feminino, não se pode deixar de ressaltar suas atividades domésticas. Ao realizar um estudo sobre saúde e trabalho de trabalhadoras de enfermagem de um hospital público, AQUINO et al. ${ }^{3}$ constataram que essas mulheres são penalizadas pelo acúmulo de trabalho doméstico, o que pode contribuir para o aparecimento de fadiga crônica, desgaste físico, além de patologias específicas.

Em relação à idade, os dados obtidos podem ser examinados na Figura 2.

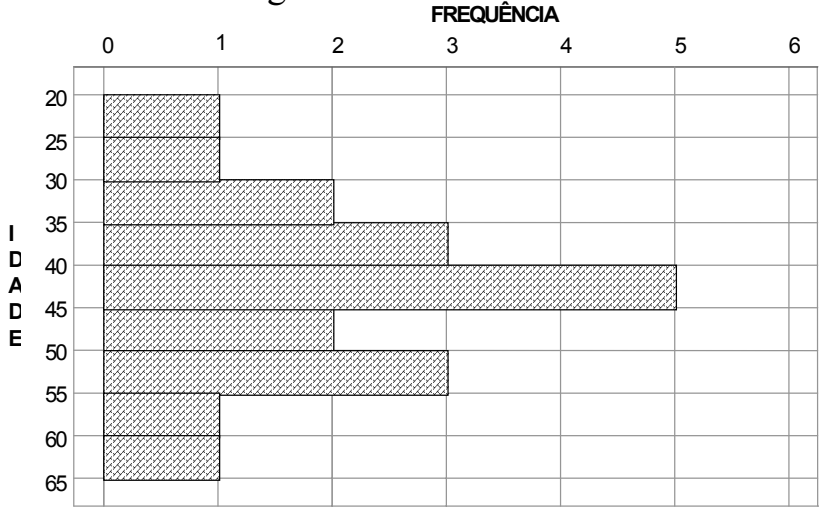

Figura 2 - Distribuição de trabalhadores de enfermagem que sofreram acidentes de trabalho relacionado com a coluna vertebral segundo a idade. Campinas, 1995 
Ainda em relação à idade, encontrou-se uma média de 43.2 anos, mediana de 42 anos, desvio padrão 10.58 , sendo a máxima de 62 e mínima de 22 anos.

Este é um fato importante na medida que o estabelecimento das causas das lesões dorsais é prejudicado pela natureza episódica dos sintomas e pelo fato de não existir um claro relacionamento temporal entre a ação física e o início dos sintomas. $\mathrm{O}$ começo da dor pode ser agudo após a ocorrência de um evento traumático ou é insidioso e associado a traumas cumulativos ${ }^{8}$. Ressalta-se também que as dores nas costas têm uma importância primordial em virtude de sua frequência e dos seus efeitos incapacitantes. São uma das principais causas de incapacidade e limitação de atividades em pessoas no período de maior produtividade ${ }^{21}$

Segundo REULER ${ }^{34}$ e WADDEL ${ }^{47}$, as algias vertebrais representam uma das causas mais comuns de limitação de atividade em pessoas com idade entre $40 \mathrm{e}$ 45 anos. $\mathrm{MC} \mathrm{ABEE}^{23}$, ao abordar a questão das dores nas costas especificamente nos trabalhadores de enfermagem, coloca que esse pessoal apresenta dores ou lesões dorsais em uma idade mais precoce. Um estudo encontrou enfermeiras de um centro médico americano com uma idade média de 32,3 anos, ao registrarem queixas de dores ou lesões nas costas no Serviço de Saúde Ocupacional ${ }^{24}$.

Esses dados apontam para a necessidade de mais estudos sobre este tema, focalizando principalmente se a injúria dorsal foi precedida por dores ou alguma patologia especificamente na coluna vertebral ou se depois deste acidente ocupacional o acidentado começa a apresentar algum tipo de problema.

A categoria profissional dos entrevistados também foi pesquisada e pode ser observada na Tabela 1 .

Tabela 1 - Proporção de trabalhadores de enfermagem que sofreram acidente do trabalho relacionado com a coluna vertebral segundo a categoria profissonal. Campinas, 1995

\begin{tabular}{lccc}
\hline CATEGORIA & $\begin{array}{c}\text { TOTAL DE } \\
\text { FUNCIONARIOS } \\
\text { Número }\end{array}$ & \multicolumn{2}{c}{ ACIDENTÁmero } \\
\hline Enfermeiro & 298 & 03 & $\mathbf{0}$ \\
Tecn.Enfermagem & 108 & 04 & 3,70 \\
Aux. Enfermagem & 614 & 10 & 1,63 \\
At. Enfermagem & 198 & 03 & 1,52 \\
\hline TOTAL & $\mathbf{1 2 1 8}$ & $\mathbf{2 0}$ & $\mathbf{1 , 6 4}$
\end{tabular}

Dos 20 acidentados, três $(15 \%)$ eram enfermeiros, quatro (20\%) técnicos de enfermagem, 10 $(50 \%)$ auxiliares de enfermagem e três $(15 \%)$ atendentes de enfermagem. Ao se realizar a proporção de acidentados com lesões dorsais em relação ao total de funcionários de suas respectivas categorias (Tabela 1 ), verifica-se que estatisticamente não foi constatada uma diferença significativa $(p=0,293)$ entre essas diferentes categorias ocupacionais.

Esses dados apontam para reforçar as evidências de que a categoria não é fator relevante para a ocorrência de acidentes. Este fato foi também constatado por SANTOS et al. ${ }^{38}$ e não coincide com outros autores que verificaram que trabalhadores com menor qualificação profissional estão mais expostos à acidentes de uma forma geral $^{41}$.

Fica difícil fazer comparações com pesquisas internacionais que focalizam especificamente as lesões nas costas em enfermagem visto que existem diversificadas categorias nos diferentes países. Geralmente os autores não definem os grupos ocupacionais estudados. Entre os autores que fizeram comparações entre a equipe de enfermagem, $\mathrm{BROWN}^{5}$; JENSEN $^{17}$ e FUORTES et al. ${ }^{11}$. encontraram que as "nursing aides" foram as mais afetadas.

A distribuição por turno pode ser analisada na Tabela 2

Tabela 2 - Proporção de trabalhadores de enfermagem que sofreram acidente do trabalho relacionado com a coluna vertebral segundo o turno de trabalho. Campinas, 1995

\begin{tabular}{lccc}
\hline \multicolumn{1}{c}{ TURNo } & $\begin{array}{c}\text { TOTAL DE } \\
\text { FUNCIONÁRIO } \\
\text { Número }\end{array}$ & \multicolumn{2}{c}{ ACIDENTADOS } \\
& Número & $\mathbf{\%}$ \\
\hline Manhã $(7-14$ horas) & 428 & 08 & 1,87 \\
Tarde $(13-20$ horas $)$ & 383 & 08 & 2,09 \\
Noite $(19-7$ horas $)$ & 407 & 04 & 0,98 \\
\hline TOTAL & $\mathbf{1 2 1 8}$ & $\mathbf{2 0}$ & $\mathbf{1 , 6 4}$ \\
\hline
\end{tabular}

Analisando-se a Tabela 2 verificou-se que não houve uma diferença estatística significante que demonstrasse um predomínio de acidentes entre os turnos de trabalho $(p=0,426)$. Esse fato pode ser explicado por questões administrativas relacionadas à ministração de determinados procedimentos básicos de enfermagem. No hospital utilizado para este estudo, determinadas técnicas como preparo do leito e higiene do paciente são divididas igualmente entre os funcionários dos três turnos das unidades de internação. Dessa forma, o período da manhã não fica sobrecarregado e não existe necessidade de se aumentar o número de pessoas trabalhando nesse horário.

Pesquisa nacionais que estudaram os acidentes do trabalho entre o pessoal de enfermagem, demonstraram que esses acidentes ocorreram principalmente no turno 
da manhã, tendo como justificativa a intensificação de atividades de assistência nesse período ${ }^{38,41}$. A questão do turno também foi investigada por MCABEE \& WILKINSON $^{24}$ que, ao realizarem um estudo com enfermeiras de um hospital de ensino americano, verificaram também uma ocorrência mais elevada de lesões dorsais no período da manhã.

Outro dado questionado foi o local que os acidentados trabalhavam quando ocorreu o acidente (Figura 3).

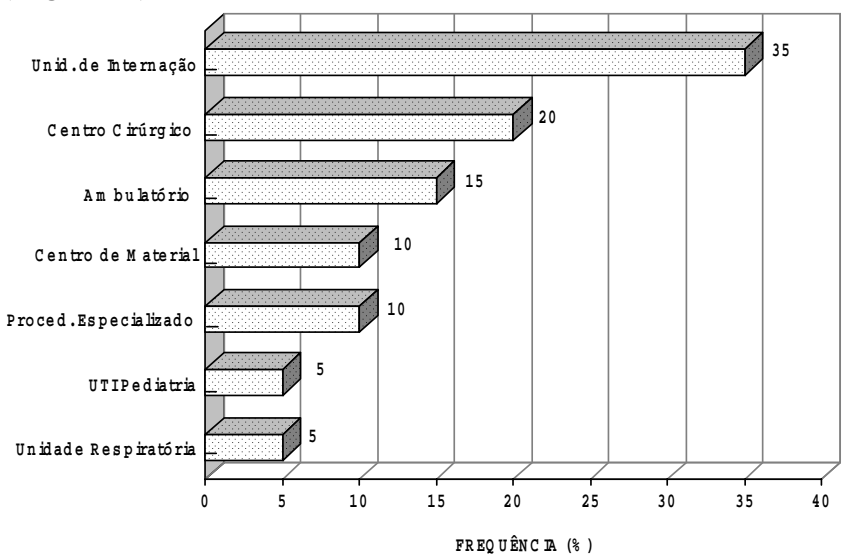

Figura 3 - Distribuição de trabalhadores de enfermagem que sofreram acidente do trabalho relacionado com a coluna vertebral segundo a unidade de trabalho, Campinas, 1995

Analisando-se a Figura 3, verifica-se que as pessoas acidentadas trabalhavam principalmente nas Unidades de Internação (35\%) e no Centro Cirúrgico $(20 \%)$. Como os acidentes relacionados com as lesões dorsais podem ocorrer nos inúmeros locais por onde transitam os funcionários de enfermagem durante suas atividades laborais, investigou-se também se o acidente ocorreu na unidade ou em outro local (Tabela 3 ).

Tabela 3 - Distribuição dos acidente do trabalho relacionado com a coluna vertebral segundo o local de ocorrência. Campinas, 1995

\begin{tabular}{lcc}
\hline L OCAL DE OCORRÊNCIA & $\begin{array}{c}\text { NÚMERO DE } \\
\text { ACIDENTES }\end{array}$ & $\mathbf{\%}$ \\
\hline 1. Na unidade de trabalho & $\mathbf{1 0}$ & $\mathbf{5 0 , 0}$ \\
- quarto (barneiro) & 2 & \\
- quarto (ao lado do leito) & 2 & \\
- corredor & 2 & \\
- posto & 1 & \\
- expurgo & 1 & \\
- sala de hemodiálise & 1 & \\
- sala de operação & 1 & \\
2. Corredores do hospital & $\mathbf{5}$ & $\mathbf{2 5 , 0}$ \\
3. Extra campus & $\mathbf{5}$ & $\mathbf{2 5 , 0}$ \\
- onibus coletivo & 3 & \\
- carro particular & 2 & \\
\hline TOTAL & $\mathbf{2 0}$ & $\mathbf{1 0 0 , 0}$ \\
\hline
\end{tabular}

A maioria dos acidentes ocorreu dentro do hospital e na própria unidade em que os funcionários estavam trabalhando. Todavia, a quantidade de acidentes que aconteceram no percurso entre a casa e o trabalho $(25 \%)$ também chama a atenção. MONTEIRO et al. ${ }^{26}$. encontraram um número semelhante $(23,40 \%)$ de acidentes de trajeto em uma pesquisa realizada entre o pessoal de enfermagem deste mesmo hospital. Estes dados apontam para a necessidade de se realizar pesquisas mais específicas sobre o acidente de trajeto que está acometendo o trabalhador hospitalar em seu deslocamento para o trabalho (violência urbana).

Todas essas informações associadas a uma descrição dos acidentes podem auxiliar na elucidação de fatores de risco que servem de subsídios para o desenvolvimento de programas de prevenção.

A Figura 4 mostra as causas dos acidentes do trabalho que lesaram as costas dos trabalhadores estudados.

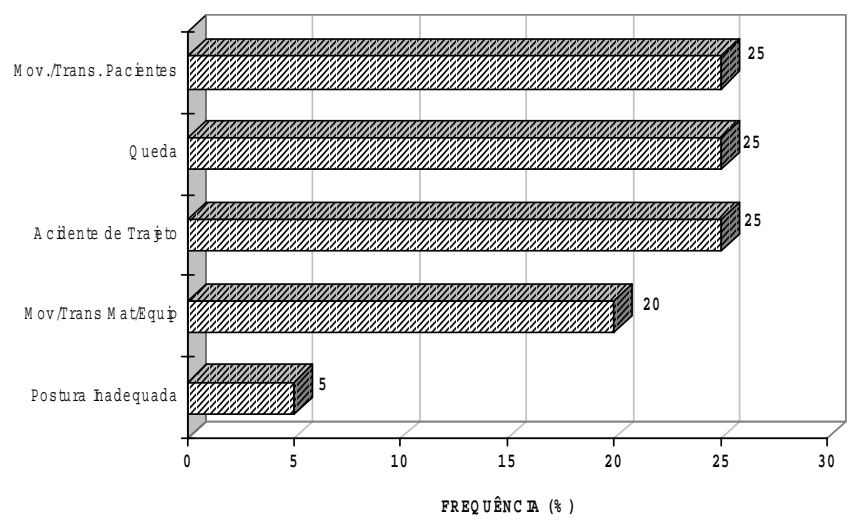

Figura 4 - Distribuição de acidentes de trabalho envolvendo a coluna vertebral em trabalhadores de enfermagem segundo as causas citadas pelos acidentados. Campinas, 1995

As respostas revelam que os acidentes geralmente ocorreram quando esses funcionários estavam movimentando ou transportando pacientes $(25 \%)$ e equipamentos $(20 \%)$ e por quedas $(25 \%)$ devido ao piso escorregadio. Os acidentes de trajeto também foram significativos $(25 \%)$.

A literatura tem demonstrado que o pessoal de enfermagem apresenta uma prevalência elevada de dores nas costas $6,7,14,43$. Todavia, existem poucos estudos que estudam especificamente a questão das lesões nas costas por acidente do trabalho.

Autores que pesquisaram as causas destas lesões em hospitais constataram que elas ocorreram principalmente pelo manuseio de pacientes, seguido pelas quedas $2,10,16,27,37,42$.

TUFFNELL ${ }^{44}$ ao estudar as causas de acidentes do trabalho entre enfermeiros de um hospital, encontrou que as lesões nas costas ocorreram principalmente ao se transportar pacientes da cama para a cadeira ou da cadeira para o banheiro. 
É importante destacar que durante a movimentação e transporte de pacientes, o peso levantado por trabalhadores de enfermagem iguala ou mesmo excede as recomendações. Além disso, são realizados sob condições desfavoráveis, com uma equipe deficiente e com equipamentos inadequados e sem manutenção ${ }^{1,9,30,44}$. Ressalta-se também que a literatura tem sugerido outros fatores de risco como falta de auxílios mecânicos, uniformes que restringem os movimentos, camas com alturas fixas, falta de preparo da equipe, entre outros $^{22,36,42}$.

Para o desenvolvimento de programas preventivos seria importante uma descrição detalhada do acidente para identificação dos fatores que o provocaram. No presente estudo, duas pessoas explicaram que tiveram que colocar o paciente de volta ao leito em uma situação de emergência.

Em relação às quedas, os sujeitos deste estudo justificaram que elas ocorreram devido ao piso escorregadio, por estar encerado ou molhado.

Ao realizar uma pesquisa sobre as algias vertebrais em funcionários de enfermagem, ALEXANDRE $^{2}$ verificou que as entrevistadas queixaram-se de dores nas costas por transportarem materiais e equipamentos tais como: monitores, respiradores, caixas com frascos de soros, galões e pesos para tração. Na presente pesquisa, dois trabalhadores declararam que sofreram lesões dorsais ao transportar torpedos de oxigênio.

HARBER et al. ${ }^{13}$, ao investigarem fatores pessoais e de trabalho associados com a ocorrência de dores nas costas entre enfermeiras de hospitais, verificaram que atividades que não envolvem o contato direto com pacientes podem ser as principais colaboradoras no desenvolvimento deste problema. Os autores também citaram que estas atividades ocupacionais são freqüentemente ignoradas em pesquisa e em programas de treinamento.

Os acidentes de trajeto quase não são mencionados em pesquisas e acredita-se que merecem um estudo específico.

Em relação as regiões da coluna vertebral mais afetadas pelos acidentes, os dados estão registrados na Figura 5.

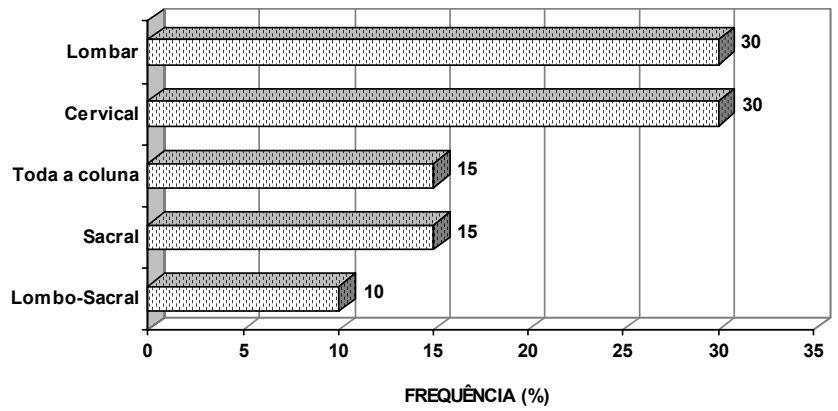

Figura 5 - Distribuição de acidente do trabalho relacionado com a coluna vertebral segundo a região afetada. Campinas, 1995
As respostas indicaram que as regiões mais atingidas foram a lombar e a cervical. Estudos biomecânicos e anatômicos esclarecem que, devido a especial mobilidade das regiões lombar e cervical, as lesões e comprometimentos da coluna vertebral nas referidas áreas são maiores que em outras, pela excessiva solicitação $^{18,19}$

Procurou-se também investigar se as pessoas acidentadas já tinham tido dores ou outras afecções relacionadas com a coluna vertebral (Figura 6).

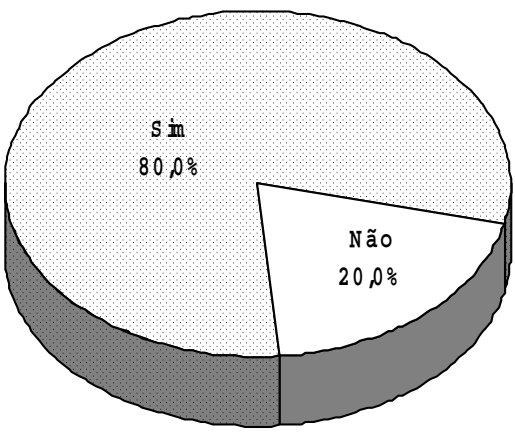

Figura 6 - Distribuição de trabalhadores de enfermagem que sofreram acidente do trabalho segundo queixa anterior de dores ou outras afecções na coluna vertebral. Campinas, 1995

A maior parte dos entrevistados afirma que já tinha tido algum tipo de problema na região dorsal. Estes achados são reforçados por autores que averiguaram que história prévia de lombalgia é um fator de risco para o desenvolvimento de injúrias dorsais ocupacionais ${ }^{15,23,37}$.

Por último, averiguou-se também que estes acidentes que lesaram a região dorsal ocasionaram um total de 489 dias perdidos de trabalho em um período de seis meses.

RAISTRICK $^{32}$ notou que durante o transcorrer de um ano, em um único distrito de saúde, foram perdidos 668 dias de trabalho devido às dores nas costas. STUBBS et $\mathrm{al}^{43}$. estimaram que 40.000 enfermeiras faltam, a cada ano, do serviço na Inglaterra, tendo também como justificativa as dores nas costas.

Uma pesquisa que abordou especificamente as lesões dorsais ocupacionais em um hospital, demonstrou que essas lesões ocasionadas por levantamentos, foram as que acarretaram os maiores custos econômicos, ocasionados principalmente pela incapacidade das pessoas retornarem ao serviço ${ }^{16}$. Ao estudar o mesmo problema em enfermeiras de um hospital americano, MCABEE \& WILKINSON ${ }^{24}$ revelaram que foram perdidos 659 dias de trabalho em um período de 32 meses.

Seria interessante que novas pesquisas acompanhassem cada caso para verificar as causas dessa permanência elevada de afastamento ao serviço.

Um aspecto que não pode deixar de ser 
mencionado é se estes acidentes do trabalho vão ser considerados legalmente como "doença ocupacional".

Determinadas posturas e movimentações adotadas por um trabalhador repetidamente durante anos podem afetar a sua musculatura e a sua constituição ósseoarticular, principalmente a da coluna e dos membros, resultando, em curto prazo, em dores que se prolongam além do horário de trabalho. A longo prazo podem resultar em lesões permanentes e deformidades músculoesqueléticas $^{4,12}$.

O fato das doenças osteomusculares poderem surgir em decorrência de um acidente específico ou aparecer lentamente com o passar do tempo dificulta o reconhecimento de sua relação com o trabalho. A predominância destes efeitos tardios impede ou dificulta que os trabalhadores estabeleçam uma relação de causa e efeito entre o acidente e as doenças ou deficiências orgânicas, muitas delas atribuídas à idade e a fatores individuais ou hereditários ${ }^{25,35}$.

Segundo o Departamento Intersindical de Estudos e Pesquisas de Saúde e dos Ambientes de Trabalho (DIESAT), para a legislação brasileira só é doença do trabalho, doença "profissional" ou "ocupacional", aquela em que é fisicamente demonstrável a relação de causa e efeito entre trabalho e doença. Ainda segundo este órgão, "esta evidência precisa ser material e materializável, carente de comprovação tanto da presença do agente no ambiente de trabalho em níveis acima dos limites de tolerância legalmente admitidos, como da sua presença e efeitos nos corpos dos trabalhadores" ${ }^{\prime 33}$.

Todos estes fatores citados ajudam a compreender a importância das afecções osteomusculares em termos de saúde ocupacional e também auxiliam a justificar porque elas têm cada vez mais despertado a atenção de profissionais da saúde em todo mundo.

\section{CONCLUSÕES}

A análise dos resultados revelou que dos 1218 trabalhadores de enfermagem de um hospital universitário, $100(8,2 \%)$ sofreram algum tipo de acidente do trabalho em um período de seis meses. Desses 100, $20(20 \%)$ estavam relacionados com lesões na coluna vertebral.

Verificou-se que esses trabalhadores que sofreram lesões eram basicamente do sexo feminino e tinham uma idade média de 43,2 anos. Não houve diferença estatística significante que demonstrasse um predomínio de acidentes entre os turnos de trabalho e entre as diferentes categorias de enfermagem envolvidas.

A maioria dos acidentes ocorreu dentro do hospital e na própria unidade em que os funcionários estavam trabalhando, quando esses trabalhadores estavam movimentando ou transportando pacientes $\mathrm{e}$ equipamentos e por quedas devido ao piso escorregadio. A quantidade de acidentes que aconteceram no percurso entre a casa e o trabalho também foi preocupante.

As respostas também indicaram que as regiões mais atingidas foram a lombar e a cervical e a maior parte dos entrevistados afirma que já tinha tido algum tipo de problema nas costas.

Todos esses dados reforçam a afirmativa de que as lesões dorsais representam um sério problema para a equipe de enfermagem merecendo novos estudos.

\section{OCCUPATIONAL ACCIDENTS AFFECTING THE SPINAL VERTEBRAE: A STUDY AMONG NURSING WORKERS OF A UNIVERSITY HOSPITAL}

In order to evaluate certain characteristics of the occurrence of occupational back injury among nursing staff, all workers affected during a period of six months were interviewed. These individuals were 43,2 years old on average and were basically female. There was no statistical significative difference showing a predominance among working turns and among the different nursing ranks involved in the accident. Most of the accidents happened within the hospital unit while they were moving or transporting patients and equipment, because of falls due to slipping floor. The most affected regions of the spinal column were the lumbar and cervical portions. The majority of the subjects affirmed that they had some type of back problem.

KEY WORDS: occupational accident, nursing workers, back injuries

\section{LOS ACCIDENTES DE TRABAJO QUE AFECTAN LA COLUMNA VERTEBRAL: UN ESTUDIO REALIZADO CON TRABAJADORES DE ENFERMERIA DE UN HOSPITAL UNIVERSITARIO}

Con el objetivo de evaluar determinadas características sobre casos de accidentes de trabajo que afectan la columna vertebral en trabajadores de Enfermería, estrevistamos todos los funcionarios que sufrieron este tipo de accidente en un periodo 
de 6 meses. Estos trabajadores eran básicamente del sexo femenino y tenían una edad média de 43,2 ãnos. No hubo diferencia estatística significante que muestre un predomínio de accidentes entre los turnos de trabajo y entre las diferentes categorías de Enfermería. La mayoría de los accidentes ocurrieron en la propia unidad de trabajo cuando esos trabajadores estaban moviendo o transportando pacientes y equipos, y por caídas debido al piso resbaladizo. Las regiones más frecuentemente afectadas fueron la región lumbar y la cervical. La mayor parte de los entrevistados afirmó que ya había tenido algún tipo de problema en la columna.

TÉRMINOS CLAVES: accidentes de trabajo, trabajadores de enfermería, lesiones en la espalda

\section{REFERÊNCIAS BIBLIOGRÁFICAS}

01. ALEXANDRE, N.M.C. Avaliação de determinados aspectos ergonômicos no transporte de pacientes. Ribeirão Preto, 1987. 114p. Dissertação (Mestrado) - Escola de Enfermagem de Ribeirão Preto, Universidade de São Paulo.

02. ALEXANDRE, N.M.C. Contribuição ao estudo das cervicodorsolombalgias em profissionais de enfermagem. Ribeirão Preto, 1993. 186p. Tese (Doutorado) - Escola de Enfermagem de Ribeirão Preto, Universidade de São Paulo.

03. AQUINO, E.M.L. et al. Saúde e trabalho de mulheres profissionais de enfermagem em um hospital público de Salvador. Rev.Bras. Enfermagem, v. 46, n 3/4, p. 245-57, 1993.

04. BARREIRA, T.H.C. Um enfoque ergonômico para as posturas de trabalho. Rev.Bras.Saúde Ocup., v. 17, n. 67 , p. 61-71, 1989.

05. BROWN, L. They don't look like weightlifters... nurses and back injury. N.Z.Hosp., v. 40, n. 7, p. 22-4, 1988.

06. CATO, C. et al. Incidence, prevalence, and variables associated with low back pain in staff nurses. AAOHN J., v.37, n. 8, p. 321-7, 1989.

07. COGGAN, C. et al. Prevalence of back pain among nurses. N.Z.Med.J., v. 107, n. 983, p. 306-8, 1994.

08. DEEDE, B.A.; MC GOVERN, P.M. Low back problems. AAOHN J., v. 35, n. 8, p. 341-8, 1987.

09. DEHLIN, O; LINDBERG, B. Lifting burden for a nursing aide during patient care in a geriatric ward. Scand J.Rehabil.Med., v.7, p. 65-72, 1975.

10. FERGUSON, D. Strain injuries in hospital employers. Med.J.Aust., v. 1, n. 8, p. 376-9, 1970.

11. FUORTES, L. J. et al. Epidemiology of back injury in university hospital nurses from review of workers' compensation records and a case-control survey. J.Occup.Med., v. 36, n. 9, 1022-6, 1994.

12. GRANDJEAN, E. Lifting the task to the man. 4. ed. London : Taylor \& Francis, 1988.

13. HARBER, P. et al. Importance of non-patient transfer activities in nursing. J.Occup.Med., v. 29, n. 12, p. 967-70, 1987.

14. HARBER, P. et al. Occupational low-back pain in hospital nurses. J.Occup.Med., v. 27, n.7, p. 51824, 1985.
15. HARBER, P. et al. Personal history, training and worksite as predictors of back pain of nurses. Am.J.Ind.Med., v. 25, n. 4, p. 519-24, 1994.

16. HOOVER, S.A. Job-related back injuries in a hospital. Am.J.Nurs., v. 73, p. 2078-9, 1973.

17. JENSEN, R. Disabling back injuries among nursing personal. Rev.Nurs.Health, v. 10, n. 1, p. 29-38, 1987.

18. KAPANDJI, I.A. Fisiologia articular. São Paulo : Manole, 1980. v. 3.

19. KHALE, W. et al. Atlas de anatomia humana. 3. ed. Rio de Janeiro : Atheneu, 1988. v.1.

20. KNOPLICH, J. As dores na coluna na medicina do trabalho. Rev.Bras.Saúde Ocup., v.8, n. 32, p. 50-2, 1980 .

21. KNOPLICH, J. A importância das dores na coluna na prática médica e na indústria. Rev.Bras.Saúde Ocup., v.9, n. 36, p. 71-4, 1981.

22. MARCHETTE, L. Back injury: a preventable occupational hazard. Orthop.Nurs., v. 4, n. 6, p. 25-9, 1985.

23. MC ABEE, R.R. Nurses and back injuries: a literature review. AAOHN J., v. 36, n. 5, p. 200-9, 1988.

24. MC ABEE, R.R.; WILKINSON, W.E. Back injuries $\&$ registered nurses. AAOHN J., v. 36, n. 3, p. 106-12, 1988.

25. MENDES, R. O impacto dos efeitos da ocupação sobre a saúde de trabalhadores. Rev.Saúde Públ., v. 22, n. 4, p. 311-26, 1988.

26. MONTEIRO, M.S.; CARNIO, A.M.; ALEXANDRE, N.M.C. Acidentes de trabalho entre o pessoal de enfermagem de um hospital universitário. Rev.Bras.Enfermagem, v. 40, n. 2/3, p. 89-92, 1987

27. NEUBERGER, J.S.; HARTLEY, S. Occupational safety and health issues affect registered nurses. Occup.Health Saf., v. 57, n. 10, p. 25-7, 1988.

28. OLIVEIRA, J. Consolidação das leis do trabalho. São Paulo: Saraiva, 1992.

29. OWEN, B.D. How to avoid that aching back. Am.J.Nurs., v. 80, n. 5, p. 894-7, 1980.

30. OWEN, B.D.; GARG, A. Reducing risk for back pain in nursing personnel. AAOHN J., v. 39, n. 1, p. 24-33, 1991. 
31. PARNIAPOUR, M. et al. Environmentally induced disorders of the musculoskeletal system. Med.Clin.North Am., v. 74,n. 2, p. 347-59, 1990.

32. RAISTRICK, A. Nurses with back pain. Nurs.Times, v. 77, n. 14, p. 853-6, 1981.

33. REBOUÇAS, A.J.A. et al. Insalubridade: morte lenta no trabalho. São Paulo: Oboré, 1989.

34. REULER, J.B. Low back pain. West.J.Med., v. 143, n. 2, p. 259-65, 1985.

35. RIBEIRO, H.P.; LACAZ, F.A.C. (org.) De que adoecem e morrem os trabalhadores. São Paulo: DIESAT, 1985.

36. RODGERS, S. Back pain. Nurs.Times, v. 81, n. 3, p. 24-6, 1985.

37. RYDEN, L.A. et al. Occupational low-back injury in a hospital employee population. Spine, v. 14, n. 3, p. 315-20, 1989.

38. SANTOS, W.D.F. et al. Acidentes típicos de trabalho em pessoal de enfermagem. Rev.Bras.Saúde Ocup., v. 17, n. 68, p. 38-42, 1989.

39. SANTOS, W.D.F.; PELÁ, N.T.R. Acidentes típicos de trabalho em pessoal de enfermagem de unidades cirúrgicas. In: JORNADA DE ENFERMAGEM EM CENTRO CIRÚRGICO, III., Ribeirão Preto, 1989. Anais. Ribeirão Preto, 1989. p. 433-42.
40. SILVA, V.E.F. Estudo sobre acidentes de trabalho ocorridos com trabalhadores de enfermagem de um hospital de ensino. São Paulo, 1988. 176p. Dissertação (Mestrado) - Escola de Enfermagem, Universidade de São Paulo.

41. SILVA, A. et al. Estudo sobre os acidentes de trabalho ocorridos com a equipe de enfermagem em unidade de centro cirúrgico de um hospital geral. In: CONGRESSO BRASILEIRO DE ENFERMAGEM EM CENTRO CIRÚRGICO, 2 , São Paulo, 1995. Anais. São Paulo, 1995. p. 94-102.

42. STUBBS, D.A. et al. Back pain research. Nurs.Times, v. 77. n. 20, p. 857-8, 1981.

43. STUBBS, D.A. et al. Back pain in the nursing profession. Ergonomics, v. 26, n. 8, p. 767-79, 1983.

44. TUFFNELL, C. Taking the strain. N.Z.Nurs J., v. 80, n. 4, p. 10-2, 1987.

45. VIDEMAN, T. et al. Low-back pain in nurses and some loading factors of work. Spine, v. 9, n. 4, p. 400-4, 1984.

46. VIDEMAN, T. et al. Patient-handling skill, back injuries and back pain. Spine, v. 14, n. 2, p. 148$55,1989$.

47. WADDEL, G. A new clinical model for the treatment of low-back pain. Spine, v. 12, n. 7, p. 632-44, 1987.

48. WORD HEALTH ORGANIZATION. Occupational hazards in hospitals. Copenhagem, 1981. (EURO Reports and Studies, 80). 